\title{
Avaliação das propriedades elétricas de barras estatóricas fabricadas com resina do tipo éter diglicidílico do bisfenol F (DGEBF) contendo nanopartículas de silica
}

\section{Evaluation of electrical properties of stator bars manufactured with bisphenol $F$ diglycidyl ether resin containing silica nanoparticles}

\author{
Rafael Novaes da Conceição ${ }^{1}$ e João Sinézio de Carvalho Campos ${ }^{1 *}$ \\ ${ }^{1}$ Faculdade de Engenharia Química, Universidade Estadual de Campinas - UNICAMP, \\ Campinas, SP, Brasil \\ *sinezio@feq.unicamp.br
}

\begin{abstract}
Resumo
O presente trabalho visa apresentar resultados da aplicação de uma resina epóxi bisfenólica (DGEBF) à base de nanopartículas de sílica $(\mathrm{RN})$ e comparar suas propriedades com a resina convencional de referência epóxi bisfenólica (DGEBA) (RE), atualmente utilizada. Neste sentido fabricaram-se protótipos de barras estatórica, destinadas a hidrogeradores, através do sistema VPI (Vácuo-Pressão-Impregnação) e avaliaram-se as propriedades elétricas pelas técnicas de fator de dissipação e envelhecimento acelerado. Dentre os resultados para as resinas observou-se que: (i) o fator de dissipação e de envelhecimento são praticamente os mesmos para ambas as resinas; (ii) o valor de tip-up resultaram em $0,014 \%$ para $\mathrm{RE}$ e $0,020 \%$ para a resina $\mathrm{RN}$ e (iii) a estimativa do tempo de vida útil esta em cerca de 40 anos, o que é aplicável para a maioria das aplicações industriais. Neste sentido sugere-se que a resina RN pode ser uma alternativa a resina RE, com um desempenho elétrico equivalente.
\end{abstract}

Palavras-chave: barras estatóricas, fita de mica, hidrogerador, nanodielétricos, nanotecnologia.

\begin{abstract}
The present work aims to present the results of an application of a bisphenolic epoxy resin (DGEBF) containing silica nanoparticles $(\mathrm{RN})$ and compare its properties with a bisphenolic epoxy resin (DGEBA) (RE), currently used. In this context, prototype stator bars for hydrogenerators were manufactured, according to the VPI (Vacuum-Pressure-Impregnation) system and their electrical properties with the tests of dissipation factor and voltage endurance. Within the results for the resins it was observed that: (i) dissipation factor and voltage endurance are practically the same for both resins; (ii) the resulting values of tip-up were $0.014 \%$ for $\mathrm{RE}$ and $0.020 \%$ for $\mathrm{RN}$ resin and (iii) the estimating of the life-time is about 40 years, what is suitable for most industrial applications. In this sense it is suggested that the RN resin can be an alternative to the RE resin, with an equivalent electrical performance.
\end{abstract}

Keywords: stator bars, mica tape, hydrogenerator, nanodielectrics, nanotechnology.

\section{Introdução}

A crescente demanda por energia elétrica tem pressionado fabricantes de hidrogeradores a desenvolverem máquinas menores e com maior eficiência de geração elétrica desde o início do século XX. A limitação de geração esbarra, entre outros fatores, nas propriedades e tecnologias de materiais compósitos isolantes disponíveis. A busca por novos materiais motiva pesquisadores em todo mundo a desenvolverem alternativas que possam suportar níveis de tensão elétrica acima de $3 \mathrm{kV} / \mathrm{mm}$, que é o padrão atual de rigidez dielétrica dos sistemas isolantes ${ }^{[1]}$. Estima-se que através de uma redução de $15 \%$ na espessura de isolação seria possível aumentar em $16 \%$ a seção transversal de cobre o que aumentaria a eficiência de um hidrogerador em $4 \% 0^{[2]}$.

A descoberta de materiais nanocompósitos isolantes (ou nanodielétricos) em escala de laboratório, com propriedades dielétricas superiores aos convencionais, despertou o interesse de diversos segmentos da indústria com seu potencial diversificado de aplicação. Resultados promissores publicados na literatura motivaram uma pesquisa neste $\operatorname{ramo}^{[3-6]}$. 
Um dos principais componentes de um hidrogerador são as barras estatóricas (ou barras Roebel), responsáveis por conduzir a energia elétrica gerada. Barras estatóricas possuem tipicamente uma seção transversal retangular composta por um feixe de condutores isolados individualmente entre si e do núcleo do estator através da isolação principal (Figura 1). A isolação principal é formada por um compósito a base de camadas de papel de mica e resina, podendo a última ser do tipo epóxi ou poliéster. A função da isolação é passiva na condução elétrica, porém fundamental para que a energia seja transportada sem perdas por descargas elétricas. Ademais garantindo a rigidez mecânica do feixe de condutores e a dissipação térmica eficiente. Um dos processos mais comumente empregados na indústria para fabricação de barras estatóricas é o processo VPI (Vácuo-Pressão-Impregnação). Tal processo consiste basicamente de uma impregnação das camadas de fita de mica sob pressão reduzida com posterior aumento da pressão. O que garante o preenchimento por resina dos vazios da fita de mica.

O presente trabalho visa testar uma resina contendo nanopartículas $(\mathrm{RN})$ previamente fabricada, que será testada como elemento de isolação no lugar da resina convencional de referência (RE), para construção de barras estatóricas - protótipos, e testes de caracterização efetuados segundo normas e/ou procedimentos industriais. A resina RE é utilizada desde a década de 60 até a presente data e é um material reconhecido no meio industrial por seus resultados de tempo de vida útil comprovados pelo tempo em operação ${ }^{[8,9]}$. A resina RN proposta foi obtida segundo estudos recentes desenvolvidos por Meichsner ${ }^{[3]}$, sem o uso de fita de mica, com resultados promissores de envelhecimento acelerado da ordem de um fator 10. A avaliação da resina RN proposta foi efetuada em comparação com as propriedades de isolação da resina RE, convencionalmente utilizada pela Voith Hydro Ltda. Tal resina tem por finalidade eliminar eventuais falhas na isolação, como por exemplo, vazios na isolação, de modo a melhorar a isolação elétrica e reduzir as perdas por transporte de energia.

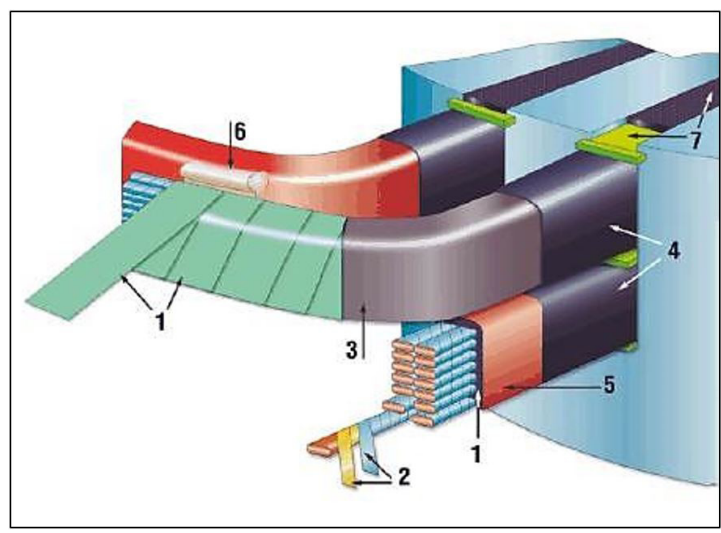

Figura 1. Desenho esquemático com os principais materiais que compõe uma barra estatórica: (1) isolação principal; (2) condutores de cobre; $(3,4)$ são os sistemas anti-corona; (5) cobertura de acabamento; (6) calço tangencial; (7) cunhas de fixação ${ }^{[7]}$.

\section{Parte Experimental}

\subsection{Preparação das resinas}

A resina utilizada para elaboração de $\mathrm{RN}$ é uma epóxi bisfenólica DGEBF na forma líquida de viscosidade 1200-1600 mPas à $25^{\circ} \mathrm{C}$, contendo nanopartículas de sílica de tamanhos entre 5 e $15 \mathrm{~nm}$, possuindo distribuição bi-modal de tamanhos de 5 a $8 \mathrm{~nm}$ e de 10 a $15 \mathrm{~nm}$. Sendo o nome comercial Epilox F16-01, fornecida pela empresa VTA $\mathrm{GmbH}$. Misturando-a com endurecedor do tipo anidrido à base de anidrido metil-hexahidroftálico, MHHPA, fornecido na forma líquida pela empresa Huntsman de nome comercial Aradur HY 1102. Após tal mistura obteve-se uma resina homogeneizada para impregnação contendo $18 \%$ em peso de nanopartículas de sílica, esta resina proposta foi denominada RN. A homogeneização foi executada utilizando um misturador tipo haste com hélices ao longo de seu comprimento, sendo que a proporção final em massa de resina e endurecedor foi de 1:1 com base no trabalho de Meichsner ${ }^{[3]}$, pois segundo este trabalho a distribuição bimodal de partículas, com tais dimensões e fração de carregamento, apresentou resultados superiores da ordem de um fator 10 em medições dos tempos de falha sob envelhecimento acelerado.

Efetuou-se a avaliação da resina proposta $\mathrm{RN}$ por comparação com os resultados de protótipos de barras estatóricas fabricadas pela empresa Voith Hydro Ltda, a qual utiliza uma resina epóxi bisfenólica. Esta resina é líquida sem modificação à base do éter diglicídilico do bisfenol $\mathrm{A}$, DGEBA, de viscosidade $4000-5500 \mathrm{mPas}$ à $25^{\circ} \mathrm{C}$, fornecida pela empresa Huntsman de nome comercial MY-790, tendo como endurecedor da resina o anidrido MHHPA (nome comercial HY-1102), ambos fornecidos pela empresa Huntsman. Mantendo-se o mesmo procedimento de preparo e a proporção 1:1 em massa entre mistura e endurecedor, esta resina será identificada neste trabalho pela sigla RE.

\subsection{Fita de mica}

A fita de mica utilizada no enfitamento das barras é composta por papel a base de mica fina $\left(160 \mathrm{~g} / \mathrm{m}^{2}\right)$, tipo MPM-NC classe 3 muscovita não calcinada ${ }^{[10]}$, depositada sobre um tecido de fibra de vidro com material aglutinante. Tal fita contém o sal de naftenato de zinco ( 0,1 a $0,4 \%$ em massa de fita) como elemento acelerador na reticulação e cura da resina.

\subsection{Barras estatóricas protótipo}

Barras protótipo foram fabricadas utilizando condutores de cobre isolados individualmente com resina híbrida de epóxi/poliéster e fibra de vidro. Tais barras apresentaram uma seção transversal de $14,5 \times 55,4 \mathrm{~mm}$ e comprimento de $2.000 \mathrm{~mm}$. Posteriormente esta barra receberá a isolação elétrica complementar.

\subsection{Preparação das barras protótipo}

As barras protótipo foram enfitadas com 10 camadas de fita de mica e $50 \%$ de sobreposição, obtendo-se uma espessura unilateral de isolação de $3 \mathrm{~mm}$, o que para esta tecnologia corresponde à uma tensão de isolação de operação de $13,8 \mathrm{kV}$. Posteriormente são inseridas em moldes e fechados e dispostas em autoclave para impregnação da 
resina, como ilustrado na Figura 2, através do processo VPI (Vácuo- Pressão-Impregnação). Fabricaram-se 18 barras com a resina contendo nanopartículas $(\mathrm{RN})$ e 14 barras com a resina convencional de referência (RE).

Antes de impregnar a resina, realizou-se o processo VPI sob as seguintes condições: colocam-se as barras estatóricas em autoclave para secagem a $65^{\circ} \mathrm{C}$, sob pressão de 0,2 mbar e durante um tempo de 10 horas, para eliminação da umidade. Após a fase de secagem transfere-se para a autoclave a resina pré-aquecida $\left(60^{\circ} \mathrm{C}\right)$, submergindo as barras com a resina. Posteriormente eleva-se a pressão no interior da autoclave para 4 bar com a injeção de $\mathrm{N}_{2}$, otimizando a penetração da resina através das camadas de fitas.

Uma vez concluído o processo VPI removem-se as barras da autoclave e submetem-nas ao processo de cura em estufa por aproximadamente 8 horas em temperatura de $140^{\circ} \mathrm{C}$. Após cura, as barras são removidas dos moldes para aplicação da proteção anti-corona em suas extremidades e então submetidas a uma segunda etapa de cura por aproximadamente 12 horas a $120^{\circ} \mathrm{C}$. Após cura da proteção anti-corona as barras estão prontas e preparadas para os ensaios elétricos. A Figura 3 resume as etapas do processo de obtenção das barras no estudo proposto com nanopartículas.

\section{Procedimentos Experimentais}

\subsection{Fator de dissipação (tan delta)}

O ensaio de fator de dissipação (tan delta) é comumente empregado como medida de controle de qualidade de produção na fabricação de barras estatóricas para avaliação da integridade da isolação e eventuais falhas decorrentes dos processos de impregnação e cura.

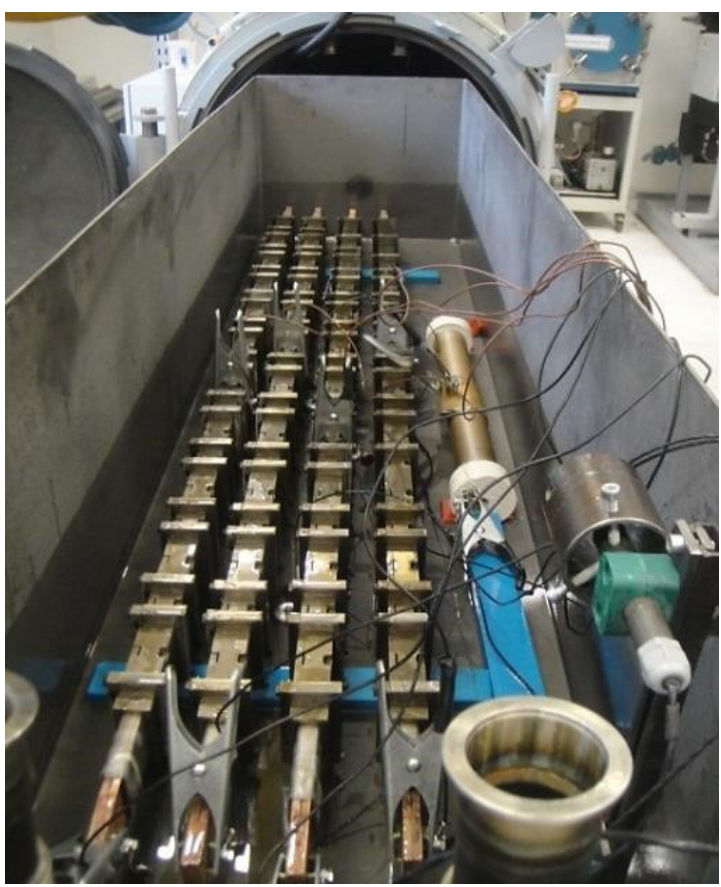

Figura 2. Banheira da autoclave com quatro barras protótipo dentro dos moldes ao fim do processo de impregnação VPI. (Cortesia Voith Hydro Ltda).
Realizaram-se ensaios do fator de dissipação em todas as barras produzidas com as resinas RN e RE conforme recomendações de ensaio especificadas pela norma IEEE $286^{[11]}$. Efetuou-se a medição de tan delta e da capacitância iniciando-se com uma tensão de $0,2 \times \mathrm{Un}(\mathrm{Un}=13,8 \mathrm{kV})$ e frequência de $60 \mathrm{~Hz}$, elevando-se a tensão em incrementos de $0,2 \times$ Un até se atingir 1,2 $\times$ Un, registrando-se a dissipação a cada incremento. Desta forma foi possível se obter o valor do tip-up entre $0,2 \times$ Un e $0,6 \times$ Un para cada medição conforme critério de aceitação em EN 50209 ${ }^{[12]}$.

Utilizando-se uma ponte de medição de tan delta da marca Tettex modelo $2805 \mathrm{HR}$, disponibilizado pela empresa Voith Hydro Ltda.

\subsection{Envelhecimento acelerado (voltage endurance test)}

Para se avaliar a expectativa de vida das barras produzidas, as mesmas foram submetidas aos ensaios de envelhecimento acelerado, que é uma prática comum utilizada pelos fabricantes de hidrogeradores. Neste ensaio é avaliado somente o aspecto elétrico, não se considerando as solicitações térmicas, mecânicas e ambientais do componente em operação.

Foram testados dois conjuntos, sendo o primeiro de 6 barras fabricadas com a resina RE e 6 com a resina $\mathrm{RN}$ sob tensão constante de $3 \times \mathrm{Un}=41,4 \mathrm{kV}$ (ou rigidez dielétrica de $13,8 \mathrm{kV} / \mathrm{mm}$ ) de acordo com norma KEMA S13/14 ${ }^{[13]}$. Cabe ressaltar que esta norma KEMA estabelece um tempo mínimo de 10 horas de sobrevivência dos componentes, ou seja, se houver falhas abaixo deste tempo o componente não é recomendado.

Testou-se um segundo conjunto de barras estatóricas seguindo as orientações da norma IEEE $1553^{[14]}$ relacionada ao ensaio de envelhecimento acelerado. Esta norma possui duas alternativas (programa A e B) em função do tempo de teste e valores de tensão elétrica. No presente trabalho optou-se pelo programa A por permitir um maior tempo de

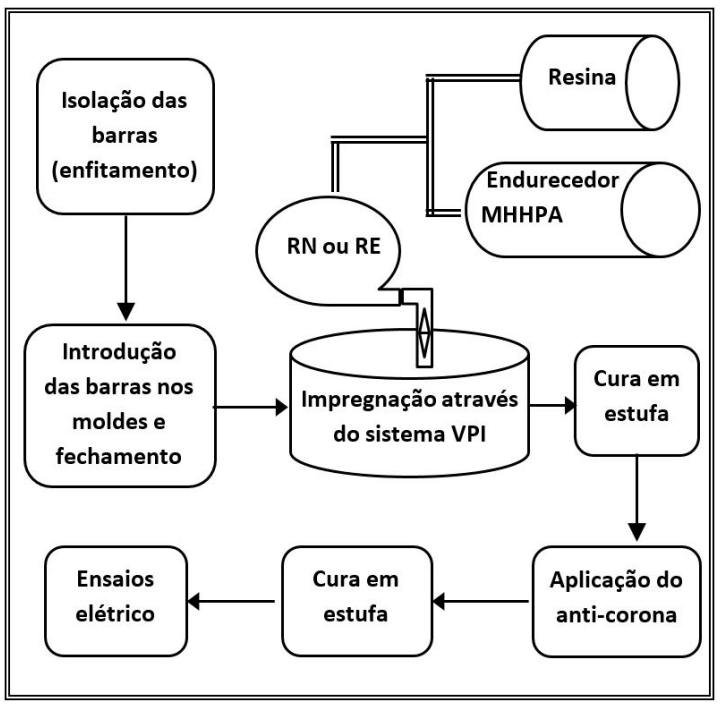

Figura 3. Fluxograma do processo utilizado para fabricação dos protótipos das barras estatóricas com resina contendo nanopartículas de sílica $(\mathrm{RN})$ ou resina convencional (RE). 
teste e um menor valor de rigidez dielétrica de $10 \mathrm{kV} / \mathrm{mm}$ em relação aos norma KEMA S13/14. Deste modo avaliando o compósito obtido em níveis de stress elétrico diferentes.

O equipamento para estes ensaios utiliza um transformador de potência marca EEAT modelo AT1001000CAAMT, com tensão e corrente ajustáveis, sendo tensão de saída 0-100 $\mathrm{kV}_{\text {ca }}$, corrente de saída $0-1000 \mathrm{~mA}_{\text {ca }}$ e $\mathrm{f}=60 \mathrm{~Hz}$, equipamento pertencente a empresa Voith Hydro Ltda e a Figura 4 ilustra tal equipamento.

\section{Resultados Experimentais}

\subsection{Fator de dissipação (tan delta)}

Os resultados obtidos nos ensaios do fator de dissipação (tan delta) em função da tensão elétrica para as barras fabricadas com a resina RE e RN podem ser visualizados na Figura 5. Para elaboração do gráfico utilizou-se a média dos resultados de fator de dissipação em cada degrau de elevação da tensão de 0,2 $\times$ Un e respectivos desvios padrão obtidos. Observa-se da Figura 5 que para ambas as resinas (RE e RN) o resultado médio do fator de dissipação é praticamente o mesmo entre 0,5 e $0,6 \%$. Nota-se também que o aumento em cada degrau de $0,2 \times$ Un resulta em um aumento muito pequeno do fator de dissipação da ordem de $0,04 \%$. Estes resultados indicam um baixo nível de perdas no dielétrico e uma boa consolidação dos compósitos nos processos de impregnação e cura ${ }^{[8]}$, ou seja, sem a formação de vazios significativos ou defeitos no compósito através da introdução de nanopartículas. Outro fator que contribuiu para uma redução nos resultados foi o uso da proteção

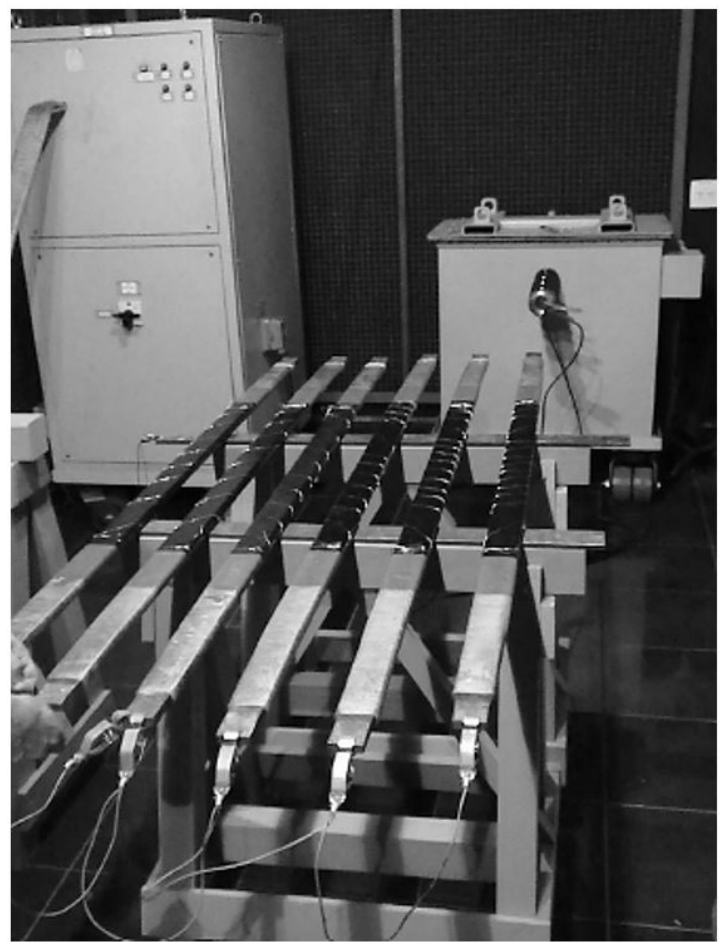

Figura 4. Equipamento para ensaio de envelhecimento acelerado das barras protótipo. anti-corona interna que reduz a distribuição da tensão entre a barra consolidada e a isolação principal ${ }^{[8]}$.

Calculando-se os valores de tip-up encontra-se 0,014\% $(\sigma=0,007)$ e $0,020 \%(\sigma=0,007)$ respectivamente para as resinas RE e RN; satisfazendo os critérios de aceitação de boa isolação, o qual de acordo com a norma EN 50209 ${ }^{[12]}$, o valor de tip-up deve ser menor que $0,25 \%$.

Não se encontrou na literatura resultados de fator de dissipação para compósitos similares aos utilizados na fabricação de barras estatóricas a base de fita de mica e resina contendo nanopartículas como no trabalho aqui proposto. Deste modo não foi possível contrapor os resultados obtidos com a literatura. Alguns artigos comparam resultados de tan delta de amostras obtidas de resina sem versus com nanopartículas variando-se a frequência de ensaio. Singha et al. ${ }^{[15]}$ realizaram ensaios de tan delta variando-se a frequência de ensaio de $1 \mathrm{MHz}$ a $1 \mathrm{GHz}$ para resinas epóxi DGEBA com nanopartículas de $\mathrm{TiO}_{2}$ e $\mathrm{Al}_{2} \mathrm{O}_{3}$ e diferentes frações de nanopartículas. Segundo os autores ao comparar-se os resultados de tan delta de um material sem nanopartículas com outro com, não se observou nenhuma diferença no resultado. Singha et al. ${ }^{[15]}$ propõem que haveriam dois processos interagindo que causariam tal comportamento. Um deles seria o número reduzido de transportadores de cargas disponíveis para condução elétrica.

A introdução de nanopartículas poderia formar um maior número de armadilhas de modo que os transportadores de carga seriam capturados por tais armadilhas. Um segundo efeito seria a introdução de interfaces e emaranhados de cadeias poliméricas no volume da matriz, causado pelas nanopartículas, que poderiam restringir a mobilidade e o deslocamento de cargas elétricas no sistema. Porém, segundo os autores uma melhor compreensão dos fenômenos interfaciais se faz necessário para uma compreensão plena dos resultados. Segundo Tanaka et al. ${ }^{[5]}$ é questionável que os resultados de tan delta possam ser reduzidos pela diminuição do tamanho das partículas (nanomização). Em alguns casos observa-se uma redução nos valores de tan delta e em outros não, e isso estaria intrinsicamente ligado à interação entre a nanopartícula e a matriz polimérica, bem como as condições de processamento.

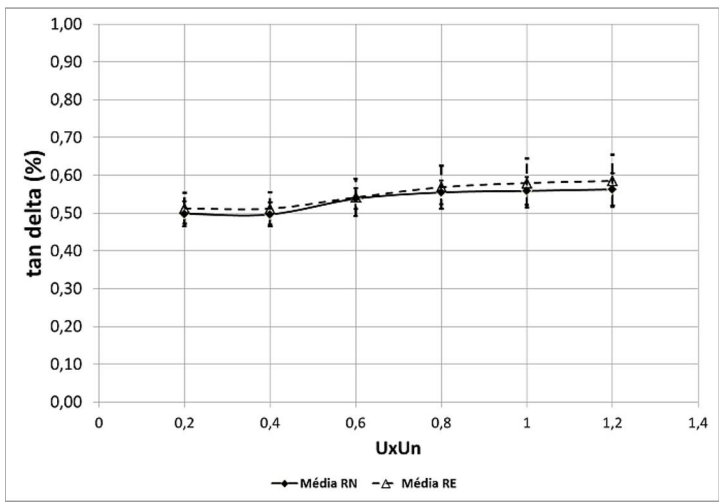

Figura 5. Resultados médios de tan delta de todas as barras fabricadas com a resina $\mathrm{RN}$ e $\mathrm{RE}$. 


\subsection{Envelhecimento acelerado (voltage endurance test)}

\subsubsection{Norma KEMA S13/S14 ${ }^{[13]}$}

A Figura 6 apresenta os resultados dos testes de envelhecimento acelerado para as barras estatóricas para as resinas $\mathrm{RN}$ e RE, onde se tem o percentual de falhas acumulado em função do tempo de ensaio. $\mathrm{O}$ valor de tensão utilizado no ensaio foi de $41,4 \mathrm{kV}$ ca e os ensaios realizados em condições ambientes $\left(28^{\circ} \mathrm{C}\right.$ e pressão de $\left.1007 \mathrm{mbar}\right)$, conforme procedimentos estipulados na norma KEMA S13/S14. Utilizou-se para o tratamento dos resultados de probabilidade de falha o método de distribuição de dois parâmetros de Weibull acumulados ${ }^{[16]}$ que é um dos métodos mais apropriados ${ }^{[17]}$ para caracterização de sistemas isolantes sólidos.

Observa-se no gráfico da Figura 6 que para ambas as resinas (RN e RE) as falhas de isolação acontecem para tempos bem superiores ao de $10 \mathrm{~h}$, sendo de $98 \mathrm{~h}$ para $\mathrm{RN}$ e de $150 \mathrm{~h}$ para RE para início de uma falha elétrica. Levando-se em consideração o valor de parâmetro de escala à $63 \%$, estabelecido pela norma IEEE 930, para o percentual de probabilidade de falha, observa-se da legenda da Figura 6 que os valores são 226 h e 198 h para a resina $\mathrm{RN}$ e RE respectivamente. Apesar do maior tempo observado de ruptura de aproximadamente $10 \%$ para resina $\mathrm{RN}$, analisando-se o gráfico, se observa que há uma sobreposição dos intervalos de confiança a $95 \%$, o que caracteriza um resultado equivalente para os dois tipos de resina. A maior dispersão dos resultados obtidos é confirmada pelo parâmetro de forma, que é inversamente proporcional à distribuição de dados, de 2,2 e 7,2 para a resina $\mathrm{RN}$ e RE respectivamente. Deste modo conclui-se que os resultados são satisfatórios frente ao critério da norma, porém não se observa nenhuma alteração com o uso de nanopartículas. Yamazaki et al. ${ }^{[18]}$ obtiveram um aumento de $8 \%$ nos resultados de tensão de ruptura para amostras de fita de mica e resina contendo nanopartículas. Os autores atribuem a melhora abaixo do esperado a uma dificuldade de difusão das nanopartículas através das camadas de mica. Esta limitação impediria uma dispersão adequada das nanopartículas na matriz polimérica

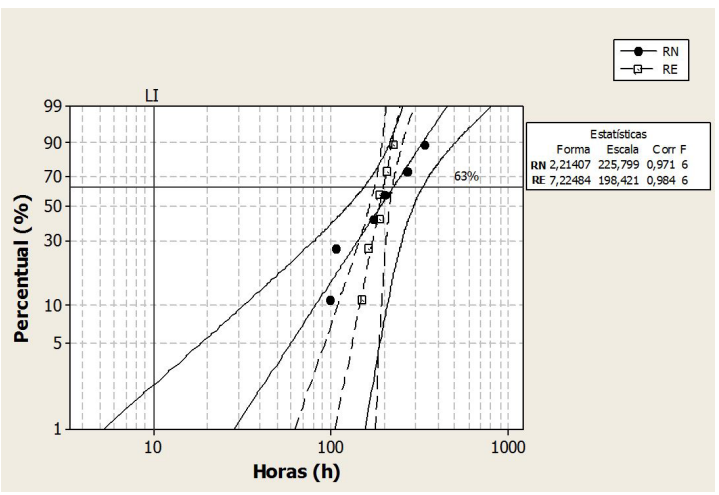

Figura 6. Probabilidade acumulada de falha em função do tempo de ensaio ou Gráfico de Weibull de envelhecimento acelerado (KEMA S13/14) para as barras produzidas com as resinas RE e RN. ( $\mathrm{LI}=10 \mathrm{~h}$, tempo limite mínimo de acordo com a norma KEMA S13/S14) e por consequência o efeito de barreira à propagação de descargas elétricas esperado.

\subsubsection{Norma IEEE $1553^{[12]}$}

Com os resultados obtidos nos ensaios de envelhecimento acelerado conforme IEE 1553A fez-se um gráfico de probabilidades acumuladas de Weibull (Figura 7). De acordo com a Figura 7 observa-se que o comportamento das resinas $\mathrm{RN}$ e RE são equivalentes e que o tempo mínimo para início de falhas é de aproximadamente $1113 \mathrm{~h}$, o que são resultados satisfatórios, uma vez que o critério de tempo mínimo é de $400 \mathrm{~h}$ segundo a norma IEEE 1553-programa A.

Como uma das barras fabricadas com a resina $\mathrm{RN}$ excedeu $3.400 \mathrm{~h}$ de ensaio sem falhas optou-se por encerrar o ensaio e utilizar o método da censura singular de acordo com a norma IEEE $930^{[14]}$ para o cálculo do parâmetro de escala. Deste modo obteve-se os valores do parâmetro de escala de $2.213 \mathrm{~h}$ e $2.292 \mathrm{~h}$ para a resina $\mathrm{RN}$ e RE respectivamente. Por este motivo que o valor de $2.099 \mathrm{~h}$ para RE apresentado na legenda da Figura 7 difere do mencionado acima.

Os valores dos parâmetros de escala e de forma obtidos para os dois tipos de resinas são iguais equivalentes, o que é comprovado graficamente pela sobreposição dos respectivos intervalos de confiança à $95 \%$. Os resultados obtidos para os ensaios de acordo com IEEE $1553^{[12]}$ confirmam a proximidade de comportamento entre os dois sistemas de resina observados também no item 4.2.1.

Alguns autores obtiveram resultados superiores de envelhecimento acelerado através da incorporação de nanopartículas. Nelson et al ${ }^{[19]}$ ao utilizarem nanopartículas de $\mathrm{TiO}_{2}$ em epóxi DGEBA conseguiram uma melhora de aproximadamente 4 ordens de magnitude em comparação com o resultado obtido com micropartículas. Segundo os autores tal incremento seria possível através de um maior controle de distribuição de cargas elétricas internas no volume do compósito e também a uma possível polarização interfacial introduzida pela enorme área interfacial das nanopartículas. Roy et al. ${ }^{[20]}$ obtiveram resultados superiores de aproximadamente 3 ordens de magnitude em polietileno com nanopartículas de $\mathrm{SiO}_{2}$ em comparação ao polietileno sem nanopartículas. Segundo IIzuka et al. ${ }^{[21]}$, os quais investigaram o envelhecimento em amostras de resina

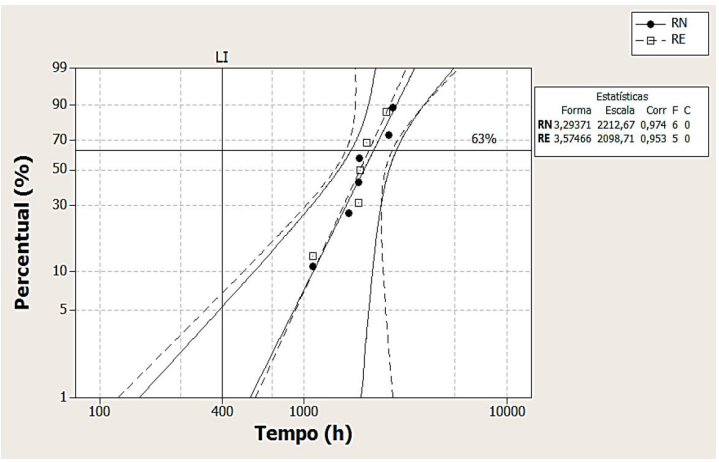

Figura 7. Gráfico obtido no ensaio de envelhecimento acelerado conforme IEEE 1553-A para as barras produzidas com as resinas em estudo. $(\mathrm{LI}=400 \mathrm{~h}$, tempo limite mínimo de acordo com a norma IEEE 1553-A). 


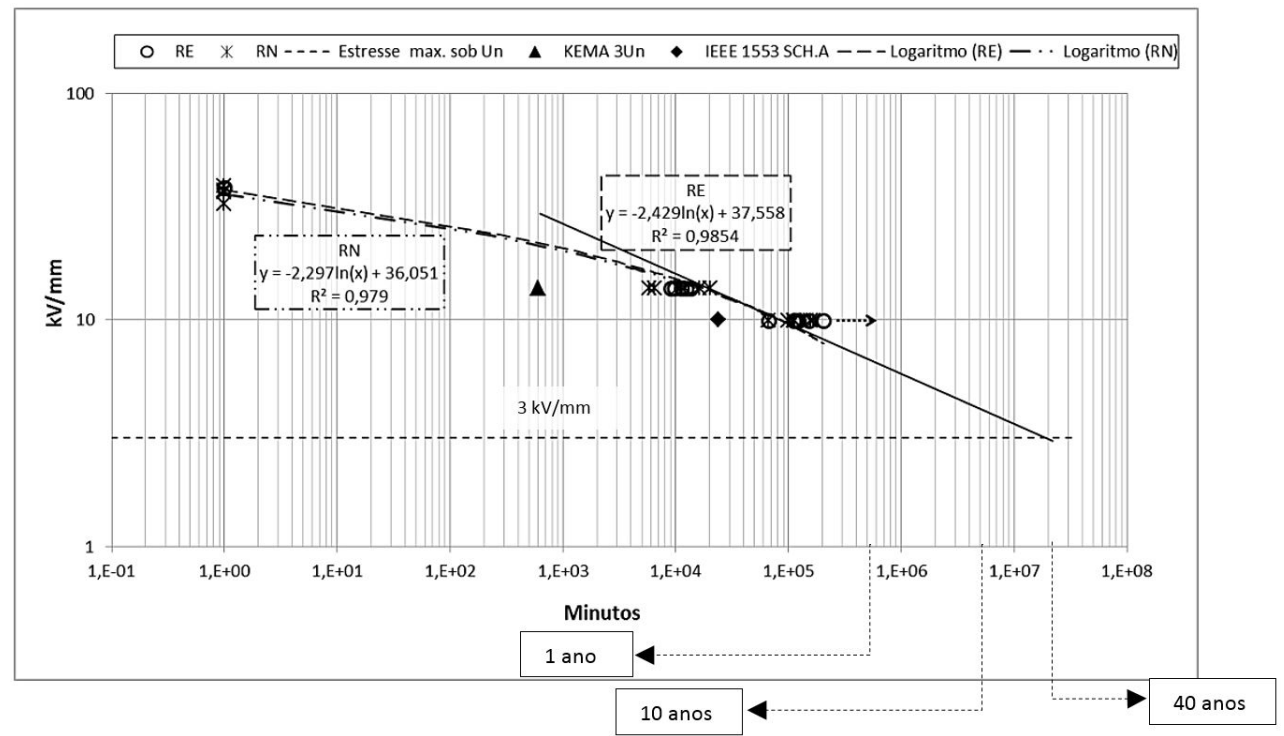

Figura 8. Rigidez dielétrica em função do tempo para as resinas RN e RE.

epóxi/sílica, explicaram que o aumento do tempo de envelhecimento esta associado a dificuldade de formação de arborecimentos na isolação, somada às fortes ligações covalentes estabelecidas entre carga e matriz, poderiam explicar os resultados superiores de envelhecimento.

Contrapondo-se os resultados obtidos nos itens 4.2.1 e 4.2.2 com os exemplos obtidos da literatura, citados anteriormente, é possível concluir-se que para a aplicação aqui proposta não houve um incremento significativo do tempo de envelhecimento acelerado em diferentes solicitações de rigidez dielétrica através do uso de nanopartículas. É possível que as propriedades dielétricas características da mica de impedimento à propagação de descargas elétricas preponderem em relação às propriedades das nanopartículas. Segundo Yamazaki et al. ${ }^{[18]}$ poderia ocorrer uma filtragem das nanopartículas pelas camadas de fita de mica de modo que estas tenham um comportamento abaixo do esperado.

\subsection{Expectativa de vida útil das barras estatóricas}

Uma estimativa complementar à distribuição de Weibull, comumente utilizada por fabricantes de hidrogeradores, é analisar o gráfico da rigidez dielétrica (tensão suportável por espessura de dielétrico) em função do tempo de ensaio, sendo estes colocados em escala logarítmica ${ }^{[1,9,22,23]}$. Realiza-se uma extrapolação considerando o valor de rigidez dielétrica para utilização industrial das barras estatóricas. Na Figura 8 são apresentados os resultados da rigidez dielétrica em função do tempo. Realizando-se uma extrapolação no tempo para um valor de $3 \mathrm{kV} / \mathrm{mm}$ para rigidez dielétrica obtém-se um tempo de vida útil aproximado de 40 anos, tempo este considerado muito satisfatório de uso deste dispositivo, o qual esta de acordo com os valores práticos observados por fabricantes ${ }^{[24]}$. Neste ensaio foi avaliado somente o aspecto elétrico, no entanto sabe-se que nas condições reais de operação o equipamento está sujeito à influência da temperatura (efeito Joule), solicitação mecânica (vibração) e condições ambientais (ozônio ${ }^{[25]}$ ), que não foram consideradas neste estudo.

Apesar dos resultados satisfatórios obtidos no presente trabalho, para as barras fabricadas com resina $\mathrm{RN}$ contendo nanopartículas de sílica, não se observa uma melhora significativa dos resultados para o fator de dissipação e envelhecimento acelerado em comparação com a resina RE. Porém, cabe ressaltar que não há na literatura trabalhos com a resina aqui utilizada e que na maioria das referências encontradas na literatura os objetos de estudo são resina epóxi pura versus resina epóxi com nanopartículas em escalas de laboratório e em nosso caso construiu-se em escala piloto (protótipo industrial).

\section{Conclusão}

Foram avaliadas as propriedades elétricas de barras protótipo fabricadas com resinas com e sem nanopartículas, RN e RE respectivamente. Pode-se concluir que: (i) os resultados de fator de dissipação e de envelhecimento são praticamente os mesmos para ambas as resinas; (ii) o valor de tip-up resultaram em $0,014 \%$ para RE e $0,020 \%$ para a resina $\mathrm{RN}$, sendo que a norma (EN $50209^{[12]}$ ) exige que seja inferior a $0,25 \%$ e (iii) a estimativa do tempo de vida útil esta em cerca de 40 anos, que é um tempo médio real para a maioria dos hidrogeradores em operação.

Assim, conclui-se que a resina epóxi bisfenólica DGEBF contendo nanopartículas de sílica pode ser uma alternativa à resina epóxi bisfenólica DGEBA, atualmente utilizada, com um desempenho elétrico equivalente.

\section{Referências}

1. Sumereder, C., \& Weiers, T. (2008). Significance of defects inside in-service aged winding insulations. IEEE Transactions on Energy Conversion, 23(1), 9-14. http://dx.doi.org/10.1109/ TEC.2006.888037. 
2. Marek, P. (2006). New carrier for high voltage insulation materials (Doctoral thesis). TU Graz, Austria.

3. Meichsner, C. (2013). Eigenschaftsoptimierung nanopartikulärer epoxidharzsysteme (Doctoral thesis). FAU Erlangen, Deutschland.

4. Tanaka, T. (2005). Dielectric nanocomposites with insulating properties. IEEE Transactions on Dielectrics and Electrical Insulation, 12(5), 914-928. http://dx.doi.org/10.1109/ TDEI.2005.1522186.

5. Tanaka, T., Montanari, G. C., \& Mülhaupt, R. (2004). Polymer nanocomposites as dielectrics and electrical insulation-perspectives for processing technologies, material characterization and future applications. IEEE Transactions on Dielectrics and Electrical Insulation, 11(5), 763-784. http://dx.doi.org/10.1109/ TDEI.2004.1349782.

6. Cao, Y., Irwin, P. C., \& Younsi, K. (2004). The future of nanodielectrics in the electrical power industry. IEEE Transactions on Dielectrics and Electrical Insulation, 11(5), 797-807. http://dx.doi.org/10.1109/TDEI.2004.1349785.

7. Von Roll, I. (2002). Electrical insulating materials: technical data sheets. Switzerland: Von Roll Isola Breitenbach.

8. Emery, F. T. (2005). Partial discharge, Dissipation factor, and corona aspects for high voltage electric generator stator bars and windings. IEEE Transactions on Dielectrics and Electrical Insulation, 12(2), 347-361. http://dx.doi.org/10.1109/ TDEI.2005.1430403.

9. Wichmann, A. (1983). Two decades of experience and progress in epoxy mica insulation systems for large rotating machines. IEEE Transactions on Power Apparatus and Systems, PAS102(1), 74-82. http://dx.doi.org/10.1109/TPAS.1983.318000.

10. International Electrotechnical Commission - IEC. (2005). IEC 60371-3-2: insulating materials based on mica. Part 3: specifications for individual materials. Sheet 2: mica paper. Geneva: IEC

11. Institute of Electrical and Electronics Engineers - IEEE. (2000). IEEE 286: recommended Practice for Measurement of Power Factor Tip-Up of Electric Machinery Stator Coil Insulation. New York: IEEE.

12. European Norm - EN. (1998). EN 50209: test of insulation of bars and coils of high-voltage machines. Brussels: EN.

13. Keuring van Elektrotechnische Materialen te Arnhem - KEMA. (2009). S13/14: KEMA: specification for hydrogen, liquid and air-cooled, synchronous a.c. generators with rated voltage 5 $k V$ and above. Arnhem: KEMA.

14. Institute of Electrical and Electronics Engineers - IEEE. (2002). IEEE 1553: trial-use standard for voltage-endurance testing of form-wound coils and bars for hydrogenerators. New York: IEEE.

15. Singha, S., \& Thomas, M. J. (2008). Permittivity and tan delta characteristics of epoxy nanocomposites in the frequency range of $1 \mathrm{MHz}-1 \mathrm{GHz}$. IEEE Transactions on Dielectrics and Electrical Insulation, 15(1), 2-11. http://dx.doi.org/10.1109/TDEI.2008.4446731.
16. Institute of Electrical and Electronics Engineers - IEEE. (2004). IEEE 930: guide for the statistical analysis of electrical insulation breakdown data. New York: IEEE.

17. Dissado, L. A. \& Fothergill, J. C. (1992). Electrical degradation and breakdown in polymers. London: Institution of Engineering and Technology.

18. Yamazaki, K., Imai, T., Ozaki, T., Cho, H., Sekiya, H., Takeuchi, M., Tanaka, M., Asayama, M., \& Osako, T. (2012). Preparation and characteristic evaluation of hydrophobic epoxy-based nanocomposites. In Annual Report Conference on Electrical Insulation and Dielectric Phenomena (CEIDP) (pp. 283-286). Montreal: IEEE. http://dx.doi.org/10. 1109/ CEIDP.2012.6378776

19. Nelson, J. K., \& Hu, Y. (2005). Nanocomposite dielectrics: properties and implications. Journal of Physics. D, Applied Physics, 38(2), 213-222. http://dx.doi.org/10.1088/00223727/38/2/005.

20. Roy, M., Nelson, J. K., Maccrone, R. K., Schadler, L. S., Reed, C. W., Keefe, R., \& Zenger, W. (2005). Polymer nanocomposite dielectrics: the role of the interface. IEEE Transactions on Dielectrics and Electrical Insulation, 12(4), 629-643. http:// dx.doi.org/10.1109/TDEI.2005.1511089.

21. Iizuka, T., Uchida, K., \& Tanaka, T. (2007). Different voltage endurance characteristics of epoxy/silica nanocomposites prepared by two kinds of dispersion methods. In Conference on Electrical Insulation and Dielectric Phenomena (pp. 236239). Vancouver: IEEE.

22. Wichmann, A., \& Gruenewald, P. (1977). Influence of dielectric stress concentration on voltage endurance of epoxy-mica generator insulation. IEEE Transactions on Dielectrics and Electrical Insulation, EI-102(6), 428-434. http://dx.doi. org/10.1109/TEI.1977.297995.

23. Tani, T., Otosaki, K., Isoma, S., Matsuda, S., \& Hirabayashi, S. (1985). Study on the voltage endurance and prediction of the dielectric breakdown of high voltage rotating machine insulations. Institute of Electrical Engineering in Japan, 105(5), 26-33. http://dx.doi.org/10.1002/eej.4391050504.

24. Stone, G. C., Boulter, E. A., Culbert, I., \& Dhirani, H. (2004). Electrical insulation for rotating machines: design, evolution, aging testing, and repair. Piscataway: Wiley-Interscience. IEEE Press Series on Power Engineering.

25. Lisevski, C. I., Wolski, C. M. O., Munaro, M., Serta, R. G., Machado, R. P., Kowalski, E., \& Pombeiro, A. (2012). Estudo do efeito do ozônio gerado durante ensaios elétricos em equipamentos de segurança confeccionados em Borracha Natural. Polímeros: Ciência e Tecnologia, 22(2), 142-148. http://dx.doi.org/10.1590/S0104-14282012005000015.

Enviado: Fev. 23, 2015

Revisado: Set. 20, 2015 Aceito: Nov. 09, 2015 